\section{Je länger sublingual, umso besser}

\section{Die gute Verträglichkeit der sublingualen Immuntherapie ermöglicht einen einfachen Behandlungsmodus ohne komplizierte Auf- dosierungsschemata. Offen ist allerdings noch die Frage, wann mit der Hyposensibilisierung begonnnen werden sollte.}

\begin{abstract}
ystematische Studien zum optimalen Beginn einer sublingualen Immuntherapie (SLIT) fehlten bisher. Erst mit Durchführung des großen Studienprogramms zur Markteinführung der Gräserallergentablette Grazax ${ }^{\circledR}$ steht ausreichend Datenmaterial zur Verfügung, um diese Frage beantworten zu können.

Ausgewertet für die Analyse wurden die Daten von drei randomisierten doppelblinden plazebokontrollierten Multizenterstudien mit verschieden langen präsaisonalen Therapiezeiträumen. Die Auswertung umfasste 934 erwachsene
\end{abstract}

Patienten mit einer durch Gräserpollen induzierten Rhinokonjunktivitis mit oder ohne leichtes bis moderates Asthma. Die 480 mit Verum Behandelten erhielten einmal täglich sublingual eine 75.000-SQ-Tablette, entsprechend etwa $15 \mu \mathrm{g}$ Phl p 5 pro Einzeldosis. Der Therapiebeginn variierte zwischen vier und 35 Wochen vor dem ersten Pollenflug. Der während der Saison erfasste Rückgang von Symptom- und Medikationsscore unter Verum im Vergleich zu Plazebo wurde ins Verhältnis gesetzt zur Dauer der präsaisonalen Behandlung.

\title{
Ozon macht Allergene sauer
}

\section{Neben einem „westlichen“ Lebensstil gilt die Luftverschmutzung als Wegbereiter der allergischen Sensibilisierung. Konkret wird unter anderem eine durch Chemikalien induzierte Veränderung von Pollen vermutet, der genaue Mechanismus ist aber noch unklar.}

$\mathbb{I}_{\text {let }}^{\mathrm{n}}$ einer Experimentalstudie wurde Pollen von Phleum pratense den wichtigsten gasförmigen Umweltverschmutzern Ozon $\left(\mathrm{O}_{3}\right)$, Stickstoffdioxid $\left(\mathrm{NO}_{2}\right)$ und Schwefeldioxid $\left(\mathrm{SO}_{2}\right)$ allein oder in Kombination ausgesetzt. Die Konzentration der Gase lag dabei im Bereich der „natürlichen“ Belastung. Nach wässriger Extraktion der Pollen wurde der Allergengehalt in einem zweidimensionalen Immunoblot mit Serum von gegen Gräserpollen allergischen Patienten ermittelt bzw. mit monoklonalen Maus-Antikörpern die Konzentration verschiedener Phleum-pratense-Antigene bestimmt.

Nach Ozonexposition konnten im Immunoblot zahlreiche chemisch veränderte Phleum-pratense-Antigene nachgewiesen werden, meist war eine verstärkt saure Reaktion der Proteine festzustellen. Eine Aziditätszunahme der Allergene ergab sich auch durch den $\mathrm{NO}_{2}-\mathrm{O}_{3}-$ Mix.

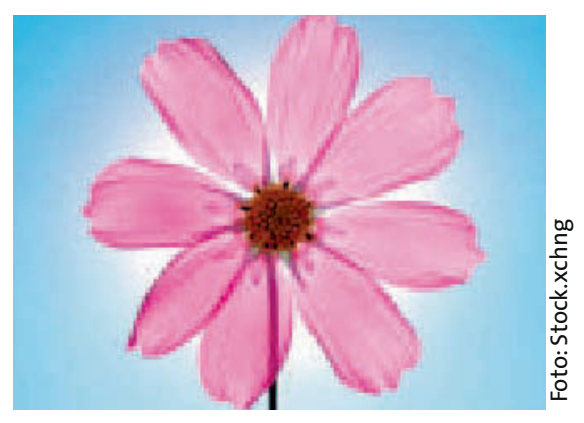

Hohe Ozonwerte im Sommer reduzieren den Allergengehalt von Pflanzenpollen.

Außerdem war die Antigenerkennung durch IgE-Antikörper bei den meisten Allergenen herabgesetzt. Die $\mathrm{NO}_{2}$ - und $\mathrm{SO}_{2}$-Exposition führte jeweils ebenfalls $\mathrm{zu}$ einer verminderten IgE-Erkennung vieler Gräserallergene, wobei die reaktiven Gase jeweils unterschiedliche Einzelallergene veränderten. Bei Begasung mit der $\mathrm{NO}_{2}-\mathrm{O}_{3}$ - oder der $\mathrm{NO}_{2}-\mathrm{SO}_{2}$-Mischung
In diesem Verum-Plazebo-Vergleich ergab sich ein signifikanter $(p<0,05)$ Rückgang von Symptom- und Medikationsscore nach einer Vorbehandlung von wenigstens acht Wochen mit einer Reduktion von 17 bzw. 23\% gegenüber Plazebo. Der Rückgang beim Symptomund Medikationsscore war umso ausgeprägter, je länger die präsaisonale Behandlung gedauert hatte $(\mathrm{p}<0,0001)$.

Fazit: Je früher eine Hyposensibilisierung mit der Gräserallergentablette Gra$\operatorname{zax}^{\circledR}$ begonnen wird, umso besser ist der in der folgenden Saison zu erwartende Therapieeffekt. Ein Minimum von acht Wochen präsaisonaler Medikation sollte nicht unterschritten werden.

$b k$

Calderon MA et al. Prolonged preseasonal treatment phase with Grazax sublingual immunotherapy increases clinical efficacy. Allergy 2007; 62: 958-61 nahm der Allergenhalt der Pollen stärker $\mathrm{ab}$ als unter Begasung mit den Einzelkomponenten.

Ursachefürden Verlust derAllergenerkennung könnte die vorzeitige Freisetzung von Allergenen. Dieser Mechanismus ist belegt, aber nur für sehr hohe Konzentrationen von Luftverschmutzergasen. Eine zweite Erklärung für die beobachteten Veränderungen könnte eine posttranslationale Modifikation der Allergenproteine sein, bei der sich unter dem Einfluss der Gase hydroxylierte Prolinstrukturen oder Disulfidbrücken ausbilden. Diese führen zusammen mit einer Proteindeglykosylierung zur Azidifizierung der Allergene und damit zu einer Veränderung ihrer IgE-Reaktivität.

Fazit: Die Exposition von Gräserpollen gegenüber gängigen Umweltgasen wie Ozon oder Stickstoffdioxid resultiert in einer herabgesetzten Allergenerkennung durch IgE-Antikörper.

Rogerieux F et al. Modifications of Phleum pratense grass pollen allergens following artificial exposure to gaseous air pollutants $\left(\mathrm{O}_{3}, \mathrm{NO}_{2}, \mathrm{SO}_{2}\right)$. Int Arch Allergy Immunol 2007; 143: 127-34 